\title{
EFICACIA DE LA INSTRUCCIÓN EN LOS ASPECTOS MECÁ- NICOS YIO SEMÁNTICOS DE LA REVISIÓN TEXTUAL
}

\author{
Olga Arlas-Gundín \\ Jesús-Nicasio García-Sánchez
}

Universidad de León

\section{RESUMEN}

En este articulo se presenta un estudio realizado sobre el proceso de revisión de la escritura mediante evaluación dinámica y el procedimiento $C D O$ con 67 estudiantes de segundo de Educación Secundaria Obligatoria. El objetivo de este trabajo es verificar que la instrucción directa en el proceso de revisión, tanto en los aspectos mecánicos como semánticos, afecta al texto re-escrito. Los estudiantes fueron asignados a una de las tres condiciones de revisión textual (revisión mecánica, revisión sustantiva, revisión mixta) y trabajaron a lo largo de nueve sesiones mediante cuatro niveles de apoyo. Los datos obtenidos muestran que el entrenamiento es efectivo, mostrando un incremento significativo en los totales de revisión. También se observa que la tarea de comparar demanda más atención y

Nota: Durante la realización de esta investigación se recibieron ayudas de la DGICYT-MEC, proyecto de investigación competitivo concedido al IP (J. N. Garcia).

Correspondencia: Universidad de León, Departamento de Psicología, Sociología y Filosofia, Ed., Psicología Evolutiva y de la Educación, Campus de Vegazana, sin, 24071-León, España, tf.: +34-987 291041 ; +34-652 8178 71; e-mail: jn.garcia@ unileon.es. 
capacidad cognitiva que la tarea de describir, siendo menor el número de revisiones realizadas a lo largo de la comparación que de la descripción.

Palabras clave: PROCESO DE REVISIÓN, PROCESO DE ESCRITURA, EVALUACIÓN DINÁMICA, PROCEDIMIENTO CDO, REVISIÓN MECÁNICA, REVISIÓN SUSTANTIVA, ASPECTOS SUPERFICIALES DE LA REVISIÓN, ASPECTOS PROFUNDOS DE LA REVISIÓN.

\section{SUMMARY}

We present an intervention study on the revising process in writing by means of dynamic assessment and CDO procedure with 67 2nd grade secondary school students. The aim of this research is to verify to what level direct instruction in the revising process, as much at a mechanical as at a semantic level, affects the re-writing of a text. The students received the instruction in their classrooms over nine sessions with four progressive levels of support. In this study, students were assigned writing compositions related with re-writing text and we included three writing revision conditions (mechanical revision, semantic revision, mixed revision). The information obtained reveals that this training is effective, showing a significant increase in the total revision measurements. Also, we observe that the comparing task demands more attention and cognitive capacity than the describing task, and that the revisions carried out during it were less than during the descriptive task.

Key words: COCAINE USE. EXPERIENTIAL AVOIDANCE DISORDER. ACCEPTANCE AND COMMITMENT THERAPY.

Los escritores expertos son los que elaboran textos más largos, de mejor calidad, con menos errores y evaluados en su estructura con mayor precisión y exactitud (Cameron, Edmundo, Wigmore, Hunt, \& Linton, 1997; Ferrari, Bouffard, \& Rainville, 1998); mientras que los escritores con menor pericia presentan un gran número de errores 
en sus composiciones, que generalmente suelen ser cortas y pobres a la vez que imprecisas. La causa principal de las diferencias que existen entre los textos elaborados por ambos tipos de escritores, debe atribuirse a una incorrecta selección de estrategias, habilidades y procedimientos por parte de los escritores menos expertos, a la vez que presentan un ínfimo uso de los procesos de autorregulación y una evaluación sin control metacognitivo (Graham, 1999a, 1999b; Wong, 1998, 1999).

Estos escritores menos expertos son los que realizan un menor número de revisiones espontáneas, haciéndose necesaria la intervención y estimulación por parte de los profesores (Perez, 2001). Este tipo de escritores consideran únicamente que deben revisar aspectos superficiales del texto como la ortografía, la puntuación, la apariencia externa...; por el contrario, los escritores expertos constantemente están revisando sus textos y atendiendo tanto a los aspectos superficiales como a los más profundos (Hacker, Plimb, Buttefield, Quathamer, \& Heineken, 1994; McCutchen, 1995).

Es importante recordar, que los diferentes modelos teóricos de la escritura entienden que el proceso de revisión es uno de los más importantes (ver revisión de Arias-Gundín \& García, 2007a) al considerar que es uno de los momentos más duros del proceso de escritura, dado que es en este instante cuando se llevan a cabo los cambios que hacen que un texto adquiera mayor calidad (Guilford, 2001; Perez, 2001). Asf, y de igual modo que en la actualidad se considera el proceso de escritura como un sistema dinámico (Rijlaarsdam \& van den Bergh, 2006) en el que influyen diversos factores cognitivos (McCutchen, 2006) prestando especial atención a la memoria operativa (Torrance \& Galraith, 2006), los modelos teóricos del proceso de revisión (Buttertield, Hacker, \& Albertson, 1996; Hayes, 2006) la consideran como una actividad recursiva a lo largo de todo el proceso de escritura estrechamente ligada con la memoria tanto operativa como a largo plazo $\theta$ influenciada por diferentes aspectos cognitivos y factores individuales (McCutchen, 2006); siendo estas algunas de las razones por las que se muestra eficaz el proceso CDO propuesto por Scardamalia y Bereiter (1985) como técnica que facilita la adquisición y ejecución de la revisión en los escritores con menor pericia (Arias-Gundín \& García, en prensa). 
Los datos obtenidos en los diferentes estudios realizados (ver revisión de Arias-Gundín \& García, 2007b) ponen de manifiesto que tanto el trabajo directo y como el entrenamiento en el proceso de revisión son eficaces. Desde que Fitzgerald y Markham (1987) estudiaron la posibilidad de que la instrucción directa en el proceso de revisión afectara tanto al conocimiento que los estudiantes tenian de dicho proceso como a su habilidad para realizar revisiones sobre el papel y a la calidad textual de los textos elaborados, son muchos los trabajos descriptivos realizados entorno a los aspectos mecánicos de la revisión (Largy, Chanquoy, \& Dèdèyan, 2004; Largy, Dèdèyan, \& Hupert, 2004)), siendo escasos los que aluden a los aspectos más profundos e semánticos de la misma (McCutchen, Francis, \& Kerr, 1997); al tiempo que los trabajos instruccionales encontrados hacen referencia, en general, al proceso de revisión (Arias-Gundín \& García, en prensa, Chanquoy, 2001, 1997; García \& Arias-Gundín, 2004; Matsumura, Petthey-Chavez, Valdés, \& Garnier, 2002), no habiéndose encontrado ninguno que trabajara de forma específica alguno de los aspectos que componen la revisión textual (aspectos mecánicos, aspectos semánticos). De esta forma, y teniendo en cuenta todo lo planteado hasta el momento, se hace necesario diseñar e implementar programas de intervención educativa que centren el trabajo bien en los aspectos mecánicos de la revisión, o en los aspectos semánticos, o por el contrario de forma conjunta en ambos.

Todo lo dicho hasta ahora justifica y enmarca la necesidad y aportación del estudio que se muestra a continuación, dado que se presenta un programa instruccional específico en el proceso de revisión de la escritura, centrado en el nivel textual de la misma; más concretamente, se presentan tres versiones del mismo programa, uno enfatizando el entrenamiento en los aspectos mecánicos de la revisión, otro en los aspectos semánticos de la misma, y por último, otro en que se trabajan por igual ambos aspectos. A lo largo de este programa se utiliza principalmente como recurso instruccional el procedimiento CDO y la evaluación dinámica.

Tomando en consideración el objetivo planteado para este estudio, que intenta conocer la eficacia de los programas instruccionales en la modificabilidad de la revisión textual utilizando la evaluación dinámica y el procedimiento CDO como recurso didáctico; la hipótesis que guía 
este estudio empírico se concreta en los siguientes términos: se espera que el programa más eficaz sea aquél que realiza una instrucción conjunta en los aspectos mecánicos y semánticos de la revisión, al considerar que los aspectos mecánicos a esta edad ya están adquiridos, y este programa les ayudará a automatizarlos, al tiempo que se trabajan de forma directa y específica en los aspectos semánticos.

\section{MÉTODO}

\section{Participantes}

En el estudio que a continuación se presenta, participaron un total de 67 estudiantes. El $52,24 \%$ de la muestra estaba constituida por mujeres $(\mathrm{N}=35)$, y el $47,76 \%$ restante eran hombres $(\mathrm{N}=32)$. Todos los estudiantes pertenecían a $2^{\circ}$ de Educación Secundaria Obligatoria, por lo que sus edades estaban comprendidas entre los 13 y 15 años, quedando su distribución como aparece en la Tabla I. Por otro lado, ninguno de los estudiantes participantes presentaba necesidades educativas especiales, estando su nivel de competencia curricular en los márgenes establecidos para $2^{\circ}$ de la E.S.O.

Tabla I. Distribución de la muestra del estudio por edades, grupo y género

\begin{tabular}{cccccc}
\hline Grupo & $\begin{array}{c}\text { Experimental } \\
\text { Meḉnica }\end{array}$ & $\begin{array}{c}\text { Experimental } \\
\text { Sustantiva }\end{array}$ & $\begin{array}{c}\text { Experimental } \\
\text { mixta }\end{array}$ & $\begin{array}{c}\text { Total } \\
\text { género }\end{array}$ \\
\hline Género & Hombres & 13 & 9 & 10 & 32 \\
& Mujeres & 10 & 14 & 11 & 35 \\
Total grupo & 23 & 23 & 21 & 67 \\
Media edad por grupo & 13,30 & 13,43 & 13,28 & \\
Edad mín-máx & $13-14$ & $13-15$ & $13-15$ & \\
\hline
\end{tabular}

\section{Diseño}

Para este trabajo se utilizó un diseño experimental con tres grupos, los cuales recibieron entrenamiento en alguno de los aspectos de la revisión textual, es decir, o en los aspectos mecánicos (revisión 
mecánica), o en los aspectos semánticos (revisión sustantiva), o bien en ambos aspectos de forma conjunta (revisión mixta).

\section{Instrumento de evaluación}

Para la evaluación de la eficacia del estudio realizado, se utilizó por un lado, el Instrumento de Revisión de la Composición Escrita: IRCE (Arias-Gundín, 2005), y por otro lado, los textos elaborados por los estudiantes en la primera y última sesión del programa, las cuales se llevaron a cabo bajo las mismas condiciones de instrucción, y cuyos resultados se presentan en este trabajo. La razón de utilizar como modo de evaluación los textos reescritos por los estudiantes en estas sesiones, no es otra que su mayor extensión en comparación con la tarea de reescritura del IRCE; al utilizar en esta evaluación un texto constituido por seis párrafos, en lugar de los tres que los estudiantes reescriben en el IRCE, nos permite analizar con mayor extensión textual las revisiones realizadas por los estudiantes en función de su localización en el texto y de la actividad demandada en cada momento. En lo que respecta a la localización en el texto, para cada una de las partes del mismo, comienzo, desarrollo y final, se elaboran dos párrafos; mientras que en la tarea del IRCE, se confecciona únicamente uno. Del mismo modo, al ser más extenso el texto elaborado en estas sesiones, se demandan cuatro actividades frente a las dos que se realizaban en el IRCE, describir y comparar, teniendo éstas en las sesiones una extensión de dos párrafos, dedicando otro párrafo tanto a la introducción como a la conclusión donde tenían que presentar su opinión personal.

Con los textos reescritos por los estudiantes en la primera y novena sesión se tomaron medidas tanto de productividad, calidad y de revisión atendiendo al tipo de operación realizada (añadir, eliminar, cambiar, reorganizar), el nivel de profundidad textual (mecánica y sustantiva), su localización (principio, desarrollo o final del texto) y el tipo de actividad que se está realizando (introducir, describir, comparar - concluir); siendo estas las mismas medidas que se recogen en la tarea de reescritura del IRCE cuya á de Cronbach es de .938, lo que implicó tener que seguir las mismas pautas de corrección. 


\section{Programa de intervención}

El programa instruccional diseñado está centrado en el trabajo específico del proceso de revisión textual mediante evaluación dinámica y la reescritura de textos. El entrenamiento se lleva a cabo en el nivel de profundidad textual de la revisión, para ello se elaboraron tres versiones del programa instruccional; una de ellas que enfatizaba el trabajo de los aspectos mecánicos de la revisión (revisión mecánica), otra que trabajaba de forma específica los aspecto semánticos de la misma (revisión sustantiva), y una tercera, que entrenaba de forma conjunta a los estudiantes tanto en los aspectos mecánicos como en los semánticos (revisión mixta).

Este programa se basa en la evaluación dinámica como principal recurso instruccional, con cuatro niveles de ayuda en la selección y ejecución de estrategias de revisión (Hayes, Flower, Schriver, Stratmen, \& Carey, 1987), como se recoge en la Figura 1. El trabajo mediante evaluación dinámica, comienza con un nivel mínimo de ayuda, es decir, directamente con la reescritura de los textos, permitiendo el trabajo en la ejecución de estrategias de la revisión mediante la ayuda de una serie de autopreguntas. A continuación se incrementa el nivel de ayuda, para realizar la reescritura de los textos mediante el apoyo de una guía de revisión, manteniendo el foco de trabajo en la ejecución de las estrategias de revisión. Aumentando progresivamente el rivel de ayuda, se centra el trabajo en la selección de la estrategia de revisión a realizar, presentando el listado completo de éstas para facilitar la elección del estudiante, para la posterior reescritura del texto. El último nivel de ayuda, es decir, el nivel máximo, aparece cuando se le presenta al estudiante el listado con todas las estrategias ejecutadas relacionadas con el texto dado para que lo reescriba. Cada nivel de ayuda se trabaja a lo largo de dos sesiones.

De modo general, se sigue la misma pauta de intervención en cada una de las sesiones. Cada sesión se inicia con la activación de los conocimientos previos relacionados con los contenidos a trabajar en dicha sesión, asI como con el afianzamiento de los conocimientos adquiridos en las sesiones previas, para ello se utilizan los ejemplos que proporcionan los propios estudiantes, aumentarido de esta manera la motivación y el interés hacia cada una de las sesiones. En un primer 
Figura 1. Niveles de ayuda utilizados en el programa instruccional de la revision textual

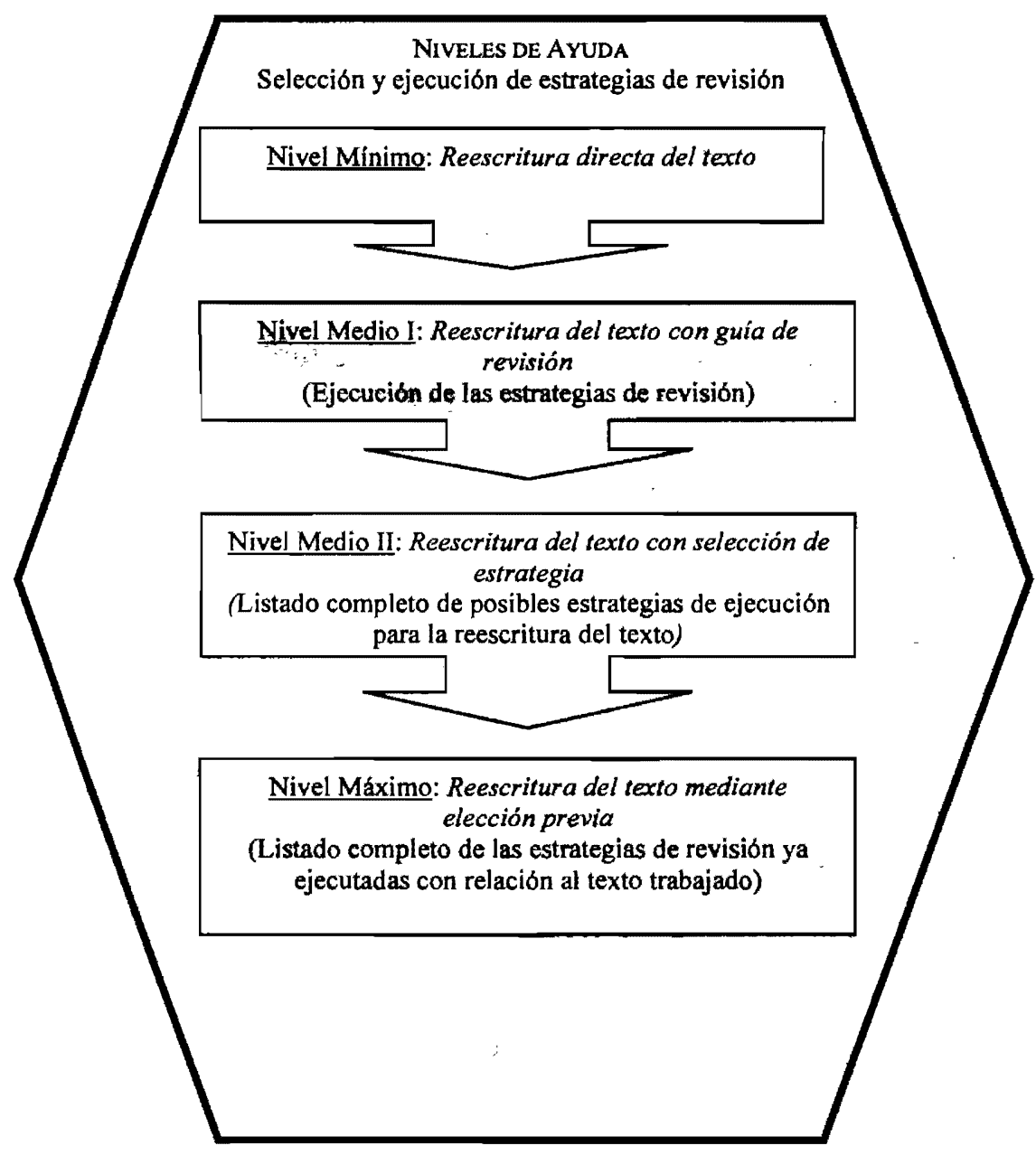

momento, la instrucción se realiza de forma conjunta a todo el grupo clase para centrar la atención de los estudiantes en los contenidos específicos que se trabajan en cada sesión. Seguidamente, tos estudiantes deben reescribir el texto correspondiente, pero antes de comenzar la tarea, el instructor les recuerda el proceso que deben seguir en su revisión: i) lectura atenta del texto, ii) detectar los errores y problemas en el texto propuesto, iii) pensar y proponer mejoras, y iv) 
reescritura del texto, es decir, deben seguir el procedimiento CDO de revisión propuesto por Scardamalia y Bereiter (1985).

En la Figura 2, se presenta de forma sintetizada la temporalización y estructuración del programa instrucional, presentando el tipo de ayuda utilizado, los aspectos trabajados y el material necesario.

Figura 2. Sintesis del programa instruccional en el proceso de revisión de la escritura mediante evaluación dinámica

\begin{tabular}{|c|c|c|}
\hline Nivel de Ayuda & Aspecto trabajado & Material \\
\hline \multirow[t]{2}{*}{$\begin{array}{c}\text { Minima } \\
(\text { Sesiones } / \text { y } 2)\end{array}$} & $\begin{array}{l}\text { Proceso de } \\
\text { escritura. }\end{array}$ & $\begin{array}{l}\text { Autopreguntas generales dirigidas a la } \\
\text { estructura y contenido global del texto. }\end{array}$ \\
\hline & $\begin{array}{l}\text { Estructura del texto } \\
\text { comparación } \\
\text { contraste. }\end{array}$ & $\begin{array}{l}\text { Autopreguntas organizadas con las mascotas } \\
\text { del proceso en cada uno de los seis párrafos } \\
\text { en que está organizado el texto. }\end{array}$ \\
\hline \multirow[t]{2}{*}{$\begin{array}{c}\text { Media I } \\
\text { (Sesiones } 3 \text { y } 4)\end{array}$} & Proceso de revisión. & $\begin{array}{l}\text { Guía de revisión, indicando únicamente las } \\
\text { categorías de que consta el tipo de revisión: } \\
\text { mecánica y sustantiva. }\end{array}$ \\
\hline & $\begin{array}{l}\text { Categorias de } \\
\text { revisión. }\end{array}$ & $\begin{array}{l}\text { Guia de revisión con autopreguntas de ayuda } \\
\text { en las categorías de que consta el tipo de } \\
\text { revisión: mecánica y sustantiva. }\end{array}$ \\
\hline \multirow[t]{2}{*}{$\begin{array}{c}\text { Media II } \\
(\text { Sesiones } 5 \text { y } 6)\end{array}$} & $\begin{array}{l}\text { Diferencia entre re- } \\
\text { escribir y corregir. }\end{array}$ & $\begin{array}{l}\text { Se presentará al estudiante una serie de } \\
\text { estrategias que puede utilizar para revisar de } \\
\text { forma global el texto; éste elegirá las que } \\
\text { considera más adecuadas y va a utilizar, } \\
\text { indicando porqué. }\end{array}$ \\
\hline & $\begin{array}{l}\text { Repaso exhaustivo } \\
\text { de las categorías de } \\
\text { revisión y las } \\
\text { diferencias entre re- } \\
\text { escribir y corregir. }\end{array}$ & $\begin{array}{l}\text { Se presentará al estudiante una serie de } \\
\text { estrategias que puede utilizar para revisar } \\
\text { cada uno de los seis párrafos de que consta el } \\
\text { texto; éste elegirá las que considera más } \\
\text { adecuadas y va a utilizar, indicando porqué. }\end{array}$ \\
\hline \multirow[t]{2}{*}{$\begin{array}{c}\text { Máxima } \\
(\text { Sesiones } 7 \text { y } 8)\end{array}$} & $\begin{array}{l}\text { Afianzamiento de } \\
\text { los conocimientos } \\
\text { adquiridos. }\end{array}$ & $\begin{array}{l}\text { Al estudiante se le presentarán tres } \\
\text { alternativas a cada uno de los seis párrafos de } \\
\text { que consta el texto que tiene que reescribir } \\
\text { para que elija el que considera que está mejor, } \\
\text { indicando porqué y reescribiendo el texto } \\
\text { presentado. }\end{array}$ \\
\hline & $\begin{array}{l}\text { A fianzamiento de } \\
\text { los conocimientos } \\
\text { adquiridos. }\end{array}$ & $\begin{array}{l}\text { Al estudiante se le presentarán tres } \\
\text { alternativas a cada una de las oraciones del } \\
\text { texto que tiene que reescribir para que elija la } \\
\text { que considera que está mejor, indicando } \\
\text { porque y reescribiendo el texto presentado. }\end{array}$ \\
\hline $\begin{array}{l}\text { Mínima } \\
\text { (Sesión 9) }\end{array}$ & $\begin{array}{l}\text { Afianzamiento de } \\
\text { los conocimientos } \\
\text { adquiridos. }\end{array}$ & $\begin{array}{l}\text { Autoprèguntas generales dirigidas a la } \\
\text { estructura y contenido global del texto. }\end{array}$ \\
\hline
\end{tabular}




\section{Procedimlento}

Tomando en consideración que el estudio que se presenta, fue llevado a cabo de forma contextualizada en tres grupo clase de $2^{2}$ de la E.S.O., la adjudicación de los grupos a cada condición del programa se realizó de forma aleatoria, sin observar el horario de la asignatura de Lengua Castellana ni el nivel educativo y rendimiento de cada una de las clases.

El programa instruccional fue diseñado para ser implementado de forma conjunta a todo el grupo clase a lo largo de nueve sesiones de unos 45-50 minutos de duración cada una de ellas.

A lo largo de cada semana, el instructor revisó y analizó cada uno de los textos reescritos por los estudiantes para poder determinar el nivel de ayuda que precisaban en la siguiente sesión.

\section{RESULTADOS}

Mediante la tarea de reescritura del IRCE se tomaron medidas de los diferentes aspectos y habilidades implicadas en la revisión. En el análisis previo realizado no se encontraron diferencias estadísticamente significativas entre los grupos al comienzo del programa $\left[\ddot{e}_{(70,210)}=.000 ; F_{(70,210)}=1.434 ; p \leqslant .409 ; c^{2}=.987\right]$.

En el análisis de la eficacia del programa instruccional, se tomaron dos tipos de medidas, una relacionada con la revisión textual y otra relacionada con la calidad y productividad del texto elaborado. Para el análisis multivariado de la varianza realizado, se utilizó un diseño factorial $3 \times 2$, siendo el factor intra-sujeto el momento de la evaluación ( $1^{z}$ y $9^{a}$ sesión) y como factor inter-sujeto el entrenamiento recibido en la revisión textual (revisión mecánica, revisión sustantiva y revisión mixta). En la Tabla II se recogen las variables totales con relación a los dos tipos de medidas tomados que muestran diferencias estadísticamente significativas.

La calidad de las composiciones escritas realizadas por los estudiantes, como se observa en la Figura 3 , se incrementa en el grupo que recibió entrenamiento específico en los aspectos semánticos de la revisión frente a aquel que lo recibió en los aspectos mecánicos; siendo también importante la mejoría sufrida por el grupo 
Eficacia de la instrucción en los aspectos...

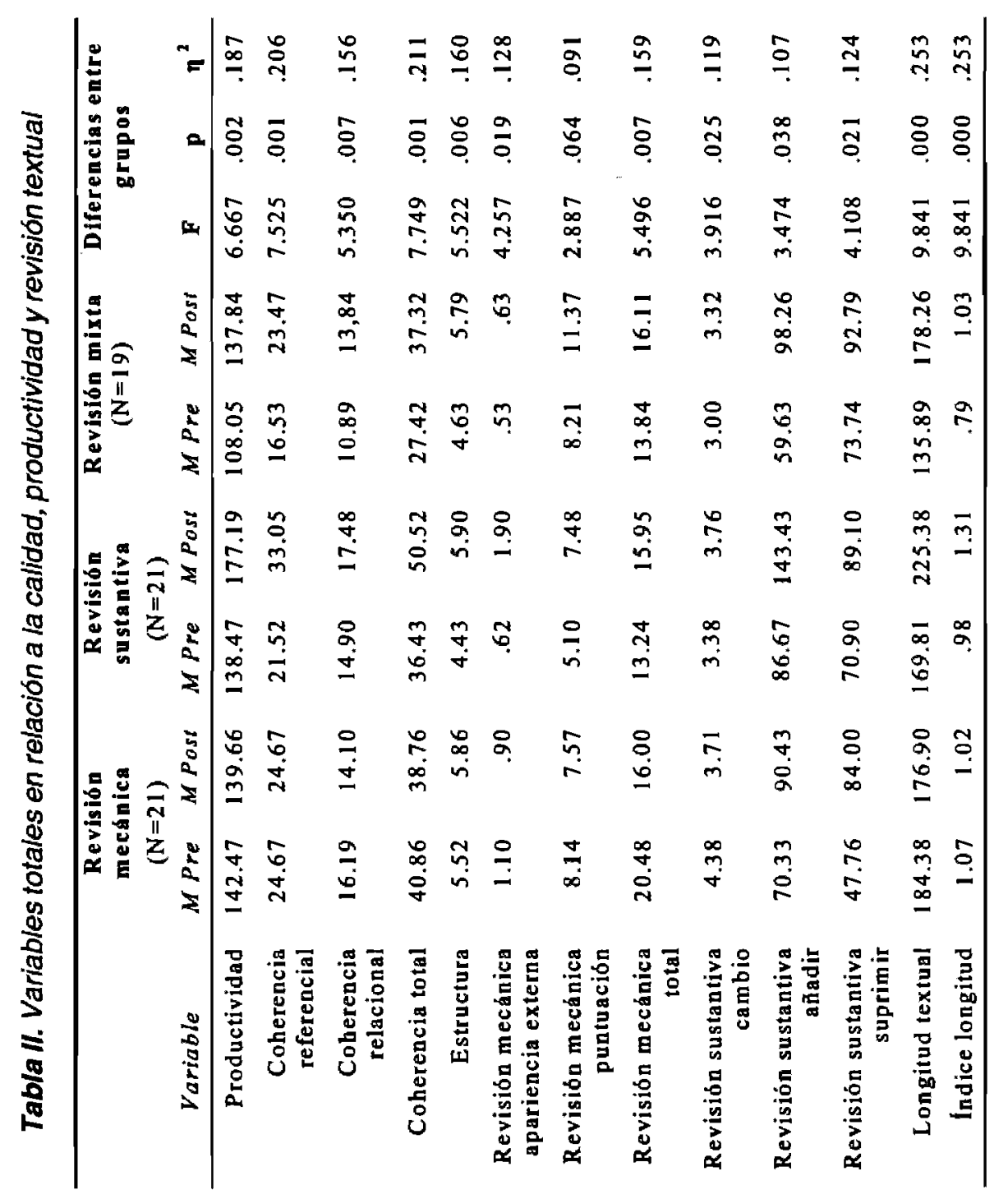

Nota: Sólo se presentan aquellos resultados estadisticamente significativos $(p \leq .05) 0$ próximos a la significación. La ç (eta al cuedrado parctal) representa et tamaño del efecto; la regla de Cohen (1988) señala: .01- .06 (efecto pequeño); .06- .14 (efecto medio); > .14 (efecto grande). 
que recibió el entrenamiento en ambos aspectos (revisión mixta). En los contrastes post hoc se evidencian diferencias estadísticamente significativas entre el grupo con entrenamiento en revisión sustantiva (S) y el de mixta (M) en coherencia referencial $\left[M_{S}=33.05 ; M_{M}=23.47\right.$; $p<.023]$, coherencia relacional $\left[M_{s}=17.48 ; M_{M}=13.84 ; p<.022\right]$ y coherencia total $\left[M_{s}=50.52 ; M_{M}=37.32 ; p<.009\right]$.

Figura 3. Calidad textual
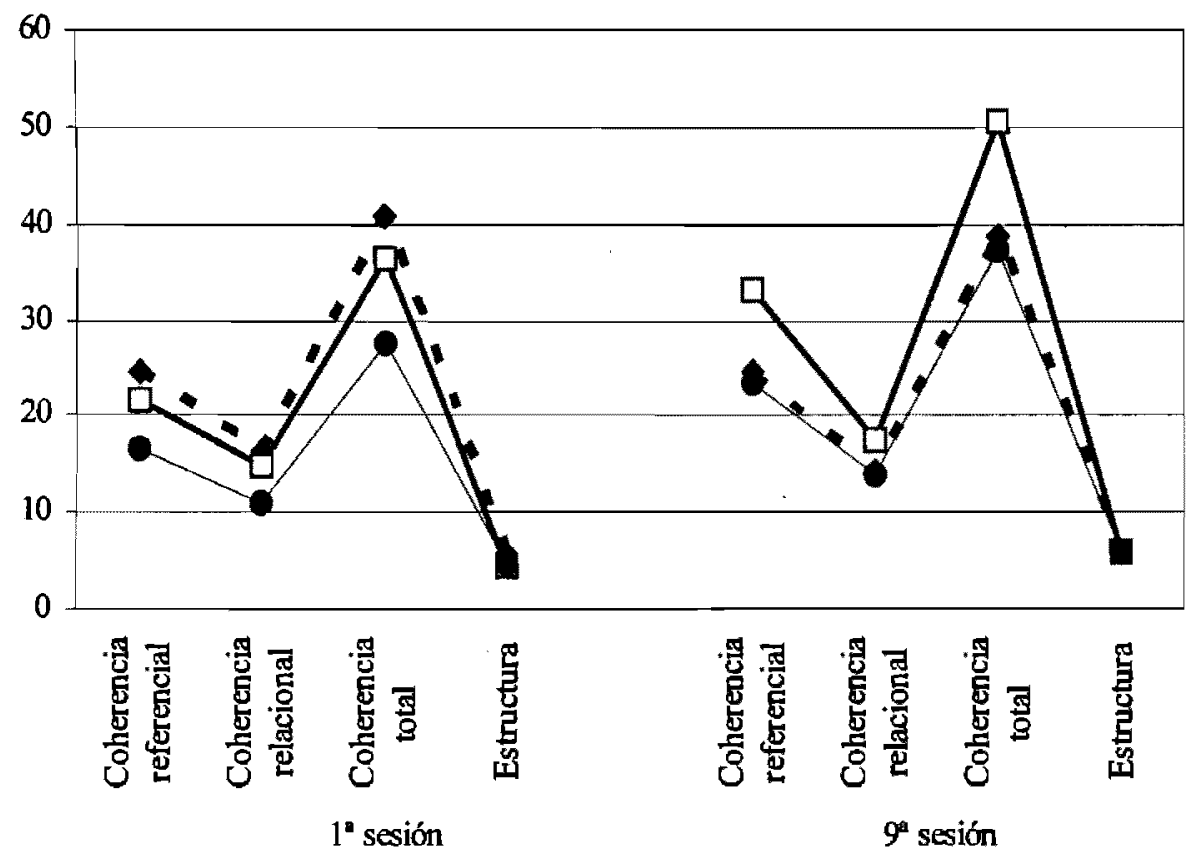

- - Mecánica - $\square-$ Sustantiva - - Mixta

Es significativo, que cuando se analizan los resultados obtenidos por los tres grupos en función del nivel de profundidad textual en donde se localizan las revisiones efectuadas por los estudiantes, respecto a la revisión a nivel mecánico, no existe ninguna diferencia entre los tres grupos; por el contrario es a nivel semántico donde se aprecian resultados interesantes, con el grupo que recibió entrenamiento específico en revisión sustantiva como el que evidencia mejores 
resultados, siendo casi iguales en los otros dos grupos, como se recoge en la Figura 4.

Figura 4. Revisiones totales en función de su profundidad textual

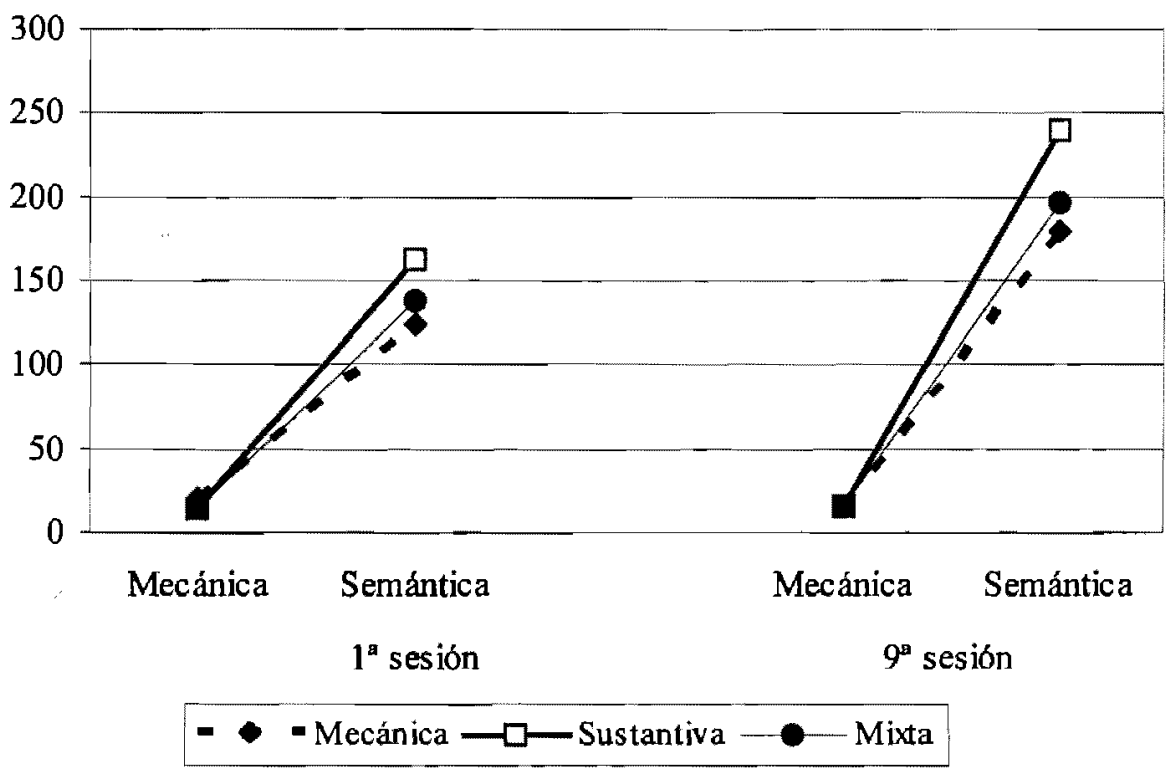

Sì realizamos un estudio más detallado de las estrategias utilizadas por los estudiantes al realizar la revisión a nivel semántico y según los contrastes post hoc realizados, se evidencian diferencias estadísticamente significativas en las estrategias tanto de añadir como de suprimir (ver Figura 5). En la estrategia de añadir las diferencias aparecen entre el grupo que recibió revisión sustantiva frente a aquellos que recibieron entrenamiento en revisión mecánica $(\mathrm{m})$ y mixta $\left[\mathrm{M}_{\mathrm{s}}=\right.$ 143.43; $\left.M_{m}=90.43 ; p \leqslant .043\right]$ y $\left[M_{s}=143.43 ; M_{M}=98.26 ; p<.039\right]$. Del mismo modo, en la estrategia de suprimir también se aprecian esta diferencias, pero en esta ocasión del grupo de revisión mecánica respecto a los otros dos grupos que recibieron entrenamiento en revisión sustantiva o mixta $\left[\mathrm{M}_{\mathrm{m}}=84.00 ; \mathrm{M}_{\mathrm{M}}=92.79 ; \mathrm{p} \leqslant .006\right]$ y $\left[\mathrm{M}_{\mathrm{m}}=\right.$ 84.00; $\left.M_{s}=89.10 ; p<.027\right]$. 
Figura 5. Estrategias de revision sustantiva

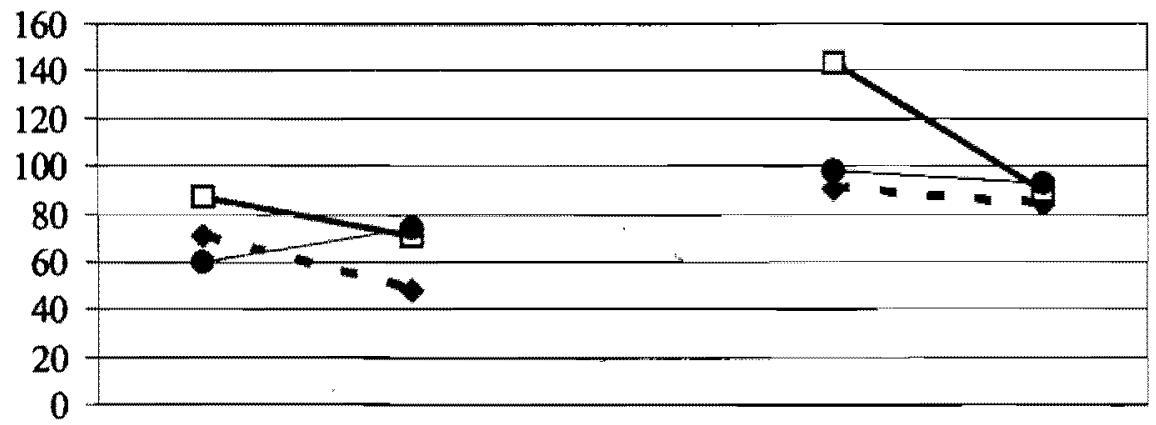

Añadir Suprimir Anadir Suprimir

$1^{\mathrm{a}}$ sesión $\quad 9^{\mathrm{a}}$ sesión

- - Mecánica $\rightarrow$ - Sustantiva - Mixta

Asimismo, las revisiones realizadas por los estudiantes a lo largo del texto, también pueden analizarse en función de su localización en el propio texto, es decir, al comienzo del mismo, en su desarrollo o al finalizarlo. En la Tabla III se recogen todas las variables que muestran diferencias estadísticamente significativas. Cabe destacar que cuando se analizaba la longitud total del texto aparecían diferencias entre los grupo $\left[F_{(1,2)}=9.841 ; p<.000 ; q^{2}=.253\right]$; por el contrario, cuando se analiza esta variable en función de la localización textual, no aparecen diferencias al comienzo del texto, siendo significativas en el desarrollo $\left[F_{(1,2)}=4.656 ; p<.013 ; q^{2}=.140\right]$ y en la finalización del mismo $\left[F_{(1,2)}\right.$ $\left.=7.940 ; p<.001 ; q^{2}=.218\right]$.

Aunque al analizar la Figura 6, se observa claramente que una vez concluida la implementación de los programas correspondientes a los respectivos grupos de trabajo existe un incremento en el numero de revisiones efectuadas por los estudiantes en todos los momentos, de forma general incrementan paulatinamente el número de revisiones que efectúan desde el comienzo de la elaboración del texto hasta su desarrollo, sufriendo un descenso considerable el número de revisiones que realizan al final del mismo; examinando los contrastes 
Eficacia de la instrucción en los aspectos...

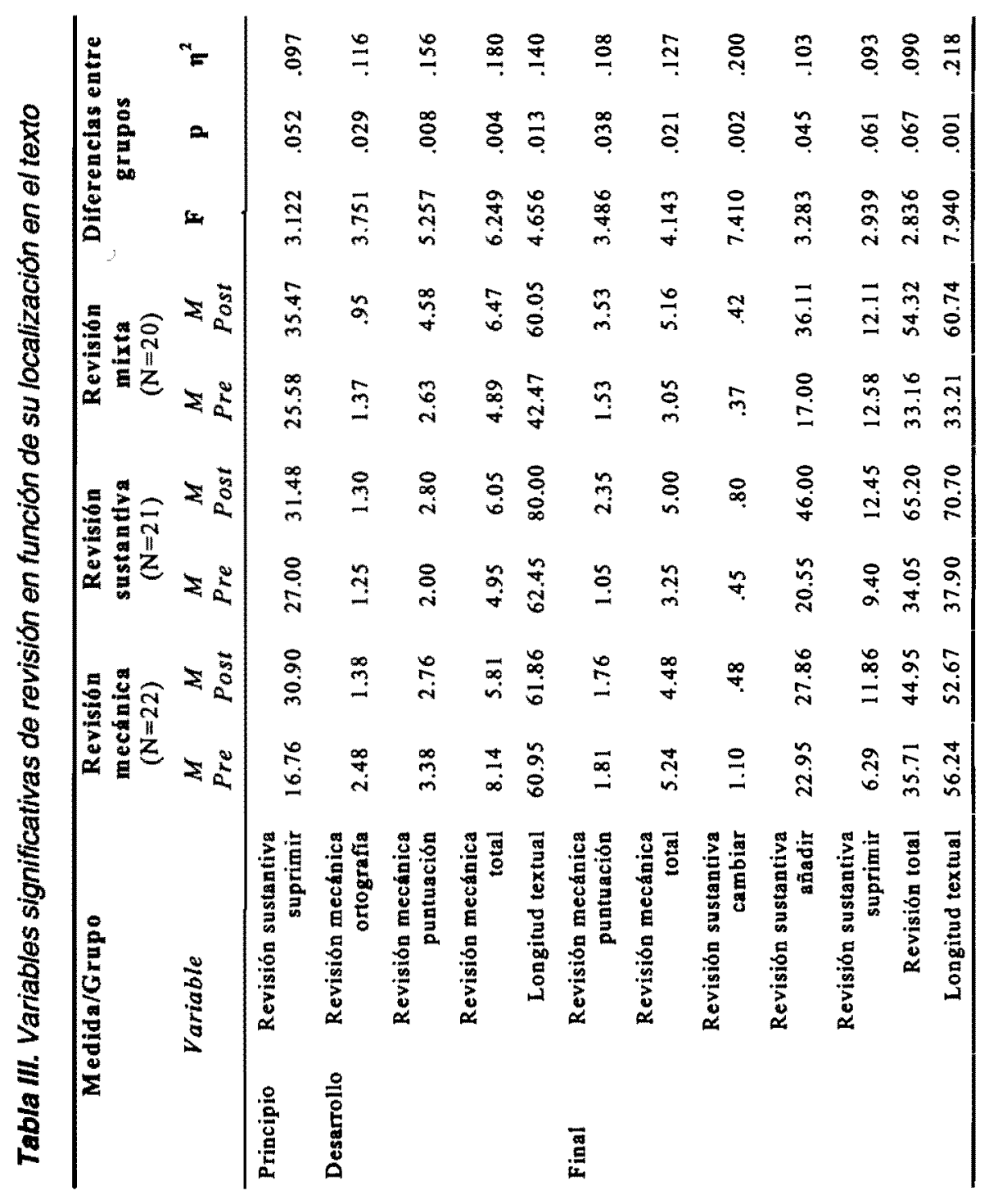

Nota: Sólo se presentan aquellos resultados estadisticamente significativos ( $p$ dw .05) 0 próximos a la significación. La $c^{2}$ (eta al cuadrado parcial) representa el tamaño del efocto; la regla de Cohen (1988) señala: .01- .06 (efecto pequeño); .06- .14 (efecto medio); > .14 (efecto grande). 
post hoc realizados, en los totales de las mismas no se observa ninguna diferencia estadísticamente significativa; por el contrario sí se observan estas diferencias en algunos de los aspectos o estrategias utilizadas. Al comienzo del texto evidencian estas diferencias en la estrategia de suprimir entre los grupos que recibieron entrenamiento en revisión mecánica y mixta $\left[M_{m}=30.90 ; M_{M}=35.47\right.$; $\mathrm{p}<066]$; entre estos dos mismos grupos, se mantienen las diferencias en el desarrollo del texto a nivel mecánico en ortografía $\left[M_{m}=1.38\right.$; $\left.M_{M}=.95 ; p<.043\right]$ y esta misma tendencia se mantiene al concluir el texto, observándose diferencias entre los mismos grupos en la estrategia de suprimir $\left[M_{m}=11.86 ; M_{M}=12.11 ; p \leqslant .063\right]$.

Figura 6. Total de revisiones según su localización en el texto

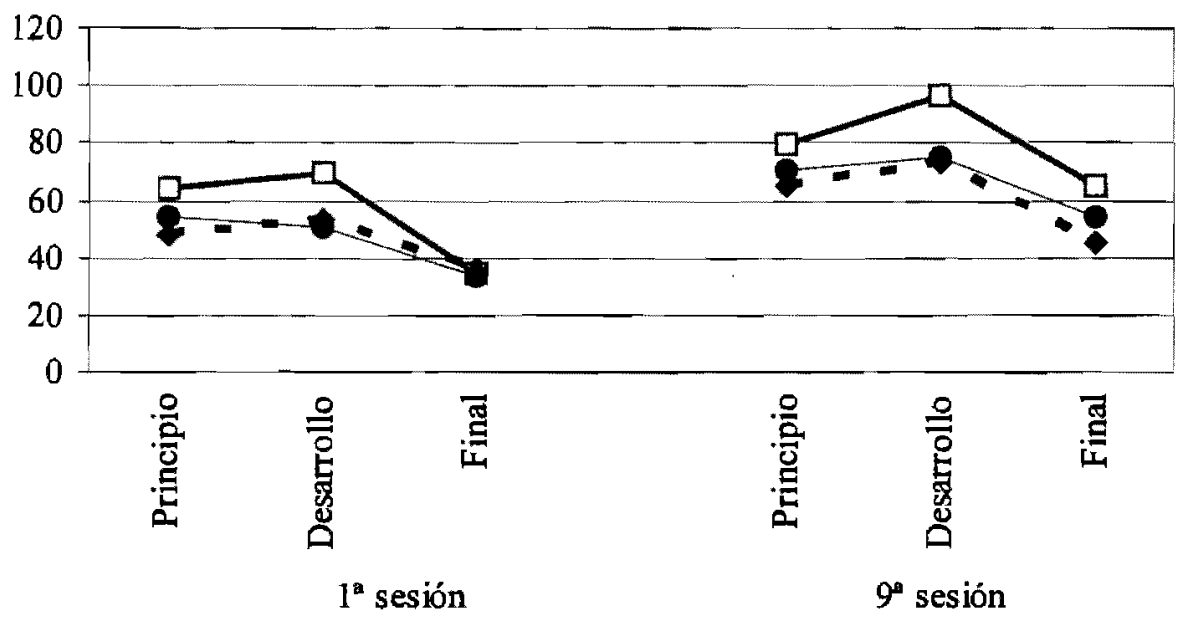

- - Mecánica $\rightarrow \square-$ Sustantiva - Mixta

Otro modo de estudiar las revisiones efectuadas por los estudiantes, es en función de la actividad que se le demanda en ese momento, es decir, introducir, describir, comparar o concluir aportando la opinión personal. En la Tabla IV se recogen todas las variables que después de los análisis multivariados de varianza realizados presentan diferencias estadísticamente significativas entre los grupos. 


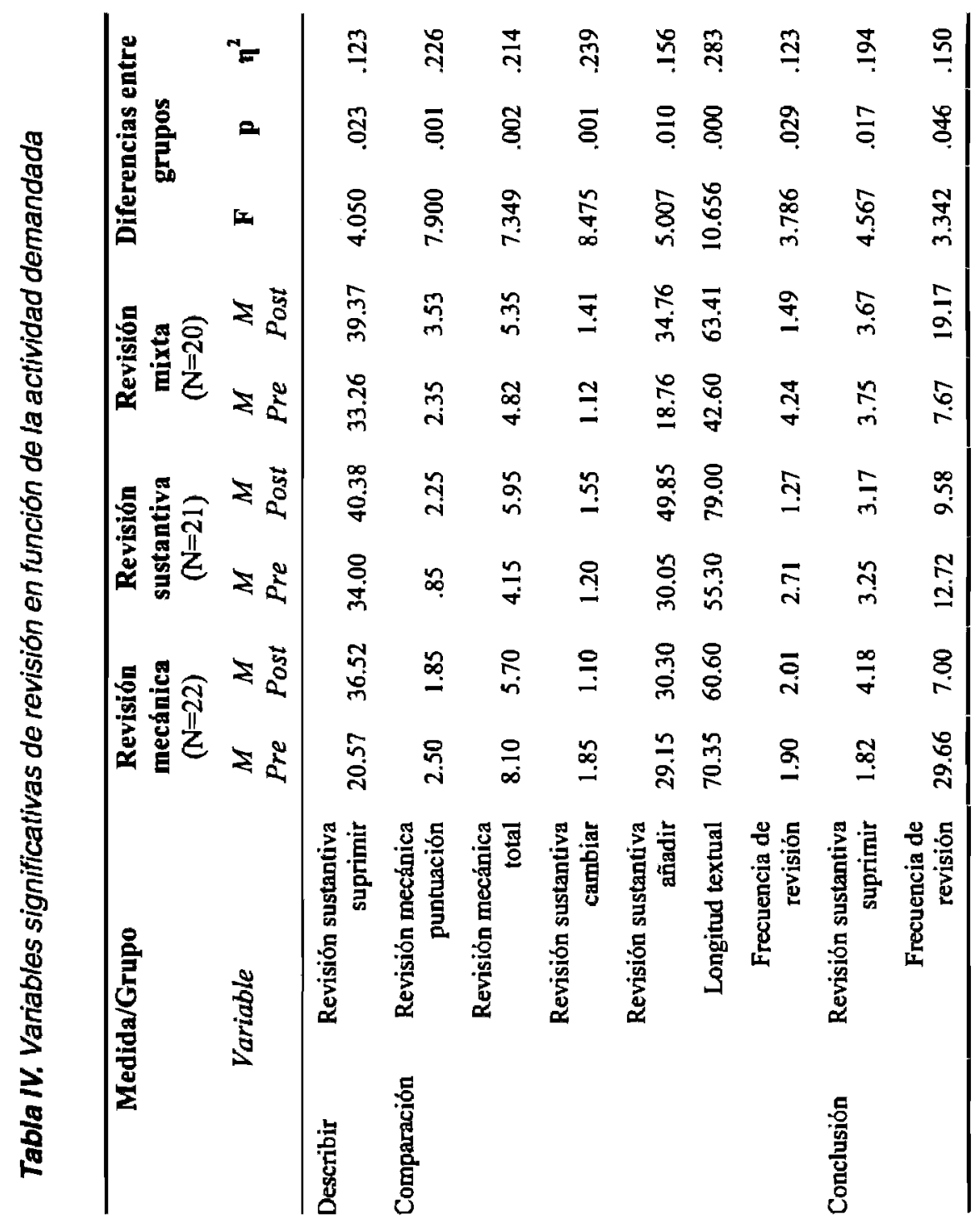

Nota: Sólo se presentan aquellos resultados estadisticamente significativos ( $p d x .05$ ) 0 próximos a la signiticación. La $\mathrm{f}^{2}$ (eta al cuadrado parcial) representa el tamaño del efecto; la regla de Cohen (1988) señala: .01- .06 (efecto pequeńo); .06- .14 (efecto medio); > .14 (efecto grande). 
Figura 7. Total de revisiones realizadas en función de la actividad demandada

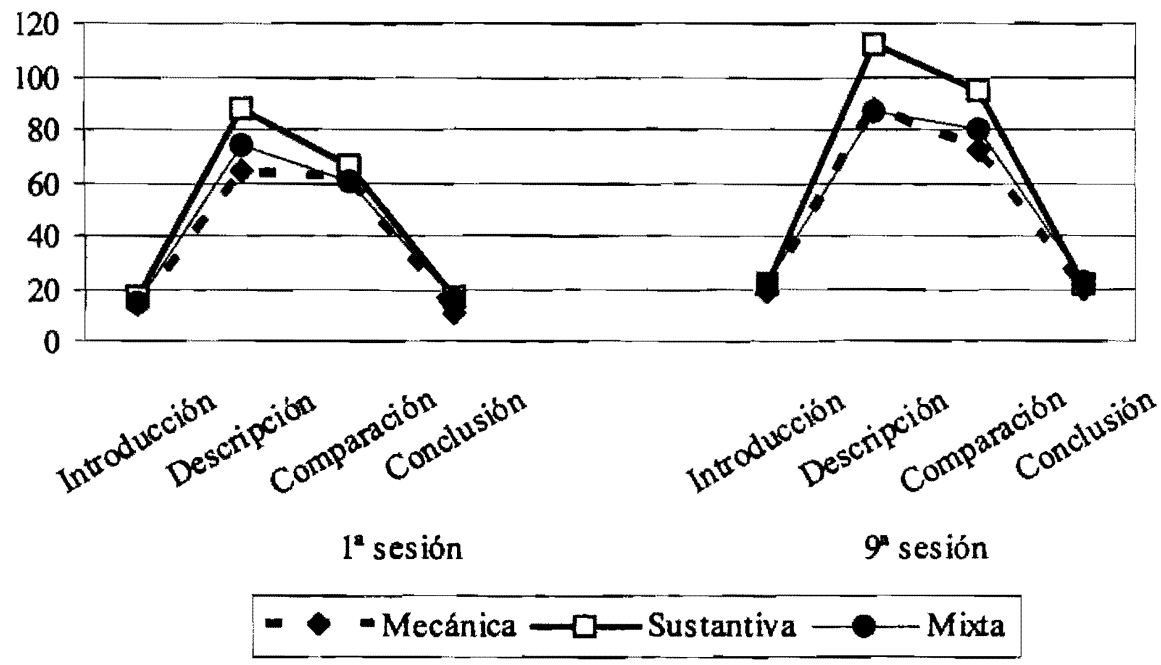

Figura 8. Revisiones mecánicas y sustantivas en función de la actividad demandada

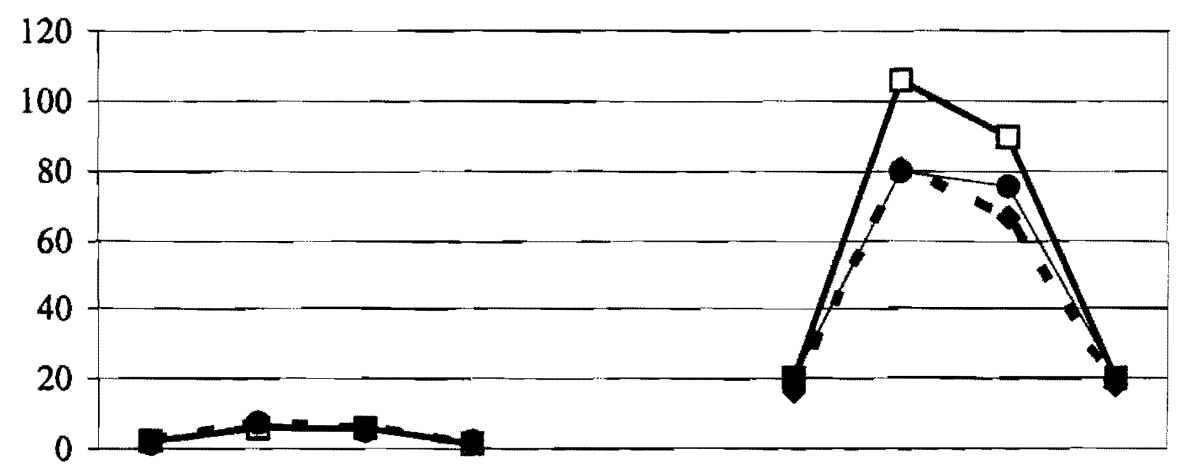

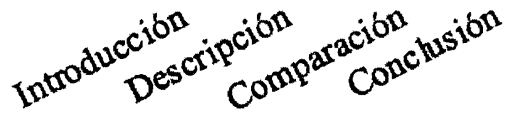

Revisiones Mecánicas

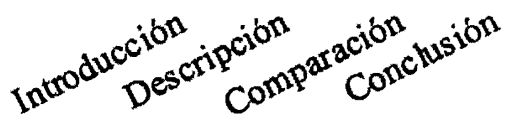

Revisiones Sustantivas 
Tal y como se recoge en la Figura 7 , son los estudiantes que recibieron entrenamiento en los aspectos semánticos de la revisión, los que incrementaron de manera notable el número de revisiones realizadas en todas las actividades demandadas.

Igual que sucede con el total de revisiones realizadas por los estudiantes en la reescritura de un texto en el número de revisiones mecánicas y sustantivas, se observa un ligero incremento de las mismas en la introducción y descripción, produciéndose un descenso progresivo en las actividades de comparar y concluir, siendo más pronunciado en la revisión sustantiva que en la mecánica, tal y como se recoge en la Figura 8.

\section{DISCUSIÓN Y CONCLUSIONES}

A la luz de los resultados obtenidos, se puede afirmar, por una parte, que los estudiantes responden positivamente a la instrucción específica en el proceso de revisión, corroborando los resultados obtenidos en trabajos previos (Conrad \& Goldstein; 1999; Garcia \& Arias-Gundín, 2004; Scott \& Vitale, 2003; Wallace et al., 1996); y dado que todos los estudiantes que participaron en una de las tres versiones del programa instruccional presentaron un incremento importante en las revisiones efectuadas, podemos concluir que estos programas diseñados en función del nivel de profundidad textual donde se producen las revisiones (mecánica, sustantiva, mixta), son eficaces en la modificación del proceso de revisión. No obstante, se esperaba que fueran más eficaces aquellos programas que no trabajaban de forma exclusiva los aspectos mecánicos de la revisión, dado que los estudiantes de educación secundaria ya deben tenerlos adquiridos (Hacker et al., 1994; Largy, Chanquoy, \& Dèdèyan, 2004; Largy, Dèdèyan, \& Hupet, 2004). De forma más específica, se suponía que la mayor eficacia la presentaría el programa de instrucción que trabajaba de forma conjunta tanto los aspectos mecánicos como semánticos de la revisión (revisión mixta), enfatizándolos por igual, al considerar que los aspectos mecánicos en este nivel educativo ya están adquiridos, y permitiría consolidarlos, al tiempo que trabajaba de forma explícita en los aspectos semánticos. En este sentido, los resultados obtenidos reflejan que existe un aumento estadísticamente 
significativo en las revisiones realizadas por los estudiantes que participaron tanto en el grupo de revisión sustantiva como en el grupo de revisión mixta frente al grupo de revisión mecánica. Sin embargo, es el grupo que participó en el programa instruccional que enfatizaba la revisión sustantiva el que muestra un mayor incremento en todos los aspectos y niveles de la revisión, con un tamaño del efecto importante.

En definitiva, se puede afirmar que en estos niveles educativos, los aspectos mecánicos de la revisión, no sólo se tienen adquiridos, sino que ya están automatizados, por lo que centrar parte de los recursos atencionales en ellos, hace que se pierda capacidad cognitiva necesaria para trabajar de forma eficaz en la revisión sustantiva, repercutiendo de esta forma en la calidad y productividad de los textos elaborados.

Se ha confirmado que la calidad y productividad de los textos elaborados han aumentado significativamente en el grupo de revisión sustantiva frente al grupo de revisión mixta, con un tamaño del efecto también elevado, lo que permite afirmar que cuando los estudiantes realizan una revisión eficaz, ésta repercute de forma relevante en el texto elaborado, lo que es coincidente con trabajos empíricos previos (Brakel, 1990; Cameron et al., 1997; Fitzgerald \& Markham, 1987; Wallace et al., 1996). No obstante, no se ha podido determinar si la eficacia en la revisión puede afectar a la estructura del texto (Brakel, 1990), dada la tipología del texto con el que se ha trabajado y su estructura ya determinada como es el caso de los textos de comparación-contraste; por lo tanto, este es uno de los aspectos a tomar en consideración para futuros trabajos.

Como ya se ha reflejado con anterioridad, ninguno de los grupos experimentó una mejora significativa en los aspectos mecánicos de la revisión; por el contrario, todos ellos sl evidenciaron un incremento significativo en sus revisiones sustantivas, manteniendo un tamaño del efecto importante. No obstante, cabe resaltar que en las dos estrategias principales desarrolladas en este nivel de la revisión, el añadir y suprimir contenido, los estudiantes del programa de revisión sustantiva elevaron de forma importante la estrategia de añadir, siendo casi imperceptible este incremento en la estrategia de suprimir; esta misma tendencia, pero de forma menos marcada presentan los 
estudiantes del programa de revisión mixta; por el contrario, esta tendencia se invierte cuando analizamos los resultados obtenidos por los estudiantes del programa de revisión mecánica; de esta forma, se puede concluir que cuando se trabaja la revisión de forma superficial y se presenta un problema textual a nivel profundo, la estrategia que seleccionan los estudiantes es eliminarlo, obteniendo resultados en la misma línea que Hayes (1996).

Cuando se analizan las revisiones realizadas por los estudiantes en función de su localización en el texto, se puede observar de forma general, que desde el comienzo del texto hasta su desarrollo el número de revisiones van incrementándose de forma paulatina, descendiendo considerablemente en la conclusión del mismo, en cualquiera de los grupos que participó en el estudio. No obstante, son significativos algunos de los resultados obtenidos. En la primera sesión, el grupo de revisión mixta mantenía una línea descendente desde el comienzo del texto en el número de revisiones efectuadas hasta su conclusión; por el contrario, esta tendencia cambia ajustándose al resto de los grupos, que realizan progresivamente un mayor número de revisiones desde el principio hasta el desarrollo del texto, disminuyendo éstas al finalizar el mismo. Del mismo modo, es significativo, que en la primera sesión todos los grupos presentaran aproximadamente un mismo número de revisiones en la conclusión del texto, mientras que en la novena se aprecian diferencias marcadas a favor del grupo de revisión sustantiva.

De lo dicho hasta este momento se puede afirma, por una parte, que la revisión es susceptible de ser entrenada respondiendo positivamente los estudiantes a ella, y por otro lado, que el proceso de revisión es una actividad muy compleja que demanda una gran atención y capacidad cognitiva que va disminuyendo a lo largo del proceso de escritura, repercutiendo de forma negativa en las revisiones realizadas (Alamargot \& Chanquoy, 2001). Así la capacidad atencional y los recursos cognitivos de los escritores son limitados, por lo que es necesario automatizar algunos procesos que permitan liberar parte de estos recursos. De esta forma, las revisiones mecánicas se automatizan en los escritores expertos, mientras que, las revisiones sustantivas interrumpen el desarrollo de otros procesos al ser estas revisiones de tipo profundo, es decir, procesos controlados. 
Para finalizar, y a la luz de los resultados obtenidos de las revisiones realizadas en función de la actividad o tarea demandada a lo largo del texto (introducción, descripción, comparación, conclusión), vuelven a confirmarse las afirmaciones realizadas hasta el momento. No se muestra ninguna diferencia a nivel mecánico entre los diferentes grupos, realizándose un mayor número de ellas en la descripción y comparación; por el contrario, existe una clara diferencia a nivel semántico, siendo siempre a favor del grupo que recibió instrucción en revisión sustantiva. No obstante, cabe destacar que el mayor número de revisiones se realizan siempre en la tarea de describir, descendiendo en la tarea de comparación y resultando escasas tanto en la introducción como en la conclusión. Una posible explicación al descenso tan brusco de las revisiones realizadas tanto en la introducción como en la conclusión, es la extensión del texto que tenían que revisar y reescribir, siendo un único párrafo en cada una de estas tareas. De esta forma, sería importante tener en consideración este aspecto para futuros trabajos; cuando el objeto del mismo sea estudiar la revisión en función de la tarea demandada a lo largo del texto, la extensión del mismo en cada una de ellas debe ser similar.

\section{REFERENCIAS BIBLIOGRÁFICAS}

Alamargot, D., \& Chanquoy, L. (2001). Through the models of writing. Kluwer Academic Puvlishers: Dordrecht.

Arias-Gundín, O. (2005). La revisión en la composición escrita para reestructurar conocimientos y aprender cambiando hábitos: desarrollo e instrucción. Tesis doctoral inédita, Universidad de León. Arias-Gundín, O., \& Garcla, J. N. (en prenșa). Instrucción en el proceso de revisión textual mediante el procedimiento $C D O$ en estudiantes que presentan bajo rendimiento. Análisis y Medicación de Conducta.

Arias-Gundín, O., \& Garcla, J. N. (2007a). Evolución del proceso de revisión en los modelos de escritura. Manuscrito enviado para su publicación.

Arias-Gundín, O., \& García, J. N. (2007b). La investigación del proceso de revisión textual en la última década. Manuscrito enviado para su publicación.

Brakel, V. L. (1990). The revising processes of sixth-grade writers with 
and without peer feedback. Joumal of educational research, 84 (1), 22-29.

Butterfield, E. C., Hacker, D. J., \& Albertson, L. R. (1996). Environmental, cognitive and metacognitive influences on text revision: Assessing the evidence. Educational Psychology Review, 8, 239-297.

Cameron, C. A., Edmunds, G, Wigmore, B., Hunt, A. K., \& Linton, M. J. (1997). Children's revision of textual flaws. International Journal of Behavioral Development, 20 (4), 667-680.

Chanquoy, L. (1997). Thinking skills and composing: Examples of text revision. En J. H. M. Hamers, \& M. Overtoom (Eds.), Inventory of European Programmes for teaching thinking (pp. 179-185). Utrecht: Sardes.

Chanquoy, L. (2001). How to make it easier for children to revise their writing: A study of text revision from $3^{\text {rd }}$ to $5^{\text {th }}$ grades. British Joumal of educational psychology, 71, 15-41.

Cohen, J. (1988). Statistical power analysis for the behavioural sciences ( $2^{\text {nd }}$ ed.). Hillsdale, NJ:LEA.

Conrad, S. M., \& Goldstein, L. M. (1999). ESL student revision after teacher-written comments: text, contexts and individuals. Joumal of second language writing, 8 (2), 147-179.

Ferrari, M., Bouffard, T., \& Rainville, L. (1998). What makes a good writer? Differences in good and poor writers' self-regulation of writing. Instructional Science. 26 (6), 473-488.

Fitzgerald, J., \& Markham, L. R. (1987). Teaching children about revision in writing. Cognition and Instruction, 4 (1), 3-24.

Garcia, J. N., \& Arias-Gundín, O. (2004). Intervención en estrategias de revisión del mensaje escrito. Psicothema, 16 (2), 194-202.

Graham, S. (1999a). The role of text production skill in writing development. Leaming Disability Quarterly. 22(2), 75-77.

Graham, S. (1999b). Handwriting and spelling instruction for students with learning disabilities: A review. Learning Disability Quarterly. 22(2), 78-98.

Guilford, W. H. (2001). Teaching peer review and the process of scientific writing. Advances in physiology education, 5 (3), 167-175. Hacker, D. J., Plumb, C., Butterfield, E. C., Quathamer, D., \& Heineken, E. (1994). Text revision: Detection and correction of errors. Joumal 
of Educational Psychology, 86 (1), 65-78.

Hayes, J. R. (2006). New directions in writing theory. En Ch. A. MacArthur, S. Graham, \& J. Fitzgerald, (Eds.), Handbook of writing research(pp.28-40). New York: The Guilford Press.

Hayes, J. R. (1996). A new framework for understanding cognition and affect in writing. En C. M. Levy, \& S. Ransdell (Eds.), The science of writing: Theories, methods, individual differences and applications (pp. 1-27). Mahwah, N.J.: Lawrence Erlbaum Associates.

Hayes, J. R., Flower, L. S., Schriver, K. A., Stratman, J. F., \& Carey, L. (1987). Cognitive processes in revision. En S. Rosenber (Ed.), Advances in applied psycholinguistics: Vol.2. Reading, writing, and language learning (pp.176-240). New York: Cambridge University Press.

Largy, P., Chanquoy, L., \& Dèdèyan, A. (2004). Ortographic revision: the case of subject-verb agreement in french. En L. Allal, $L$. Chanquoy, \& P. Largy (Eds.), Revision cognitive and instructional processes (pp. 39-62). New York: Kluwer Academic Publishers.

Largy, P., Dédéyan, A., \& Hupet, M. (2004). Ortgraphic revision: a developmental study of how revisers check verbal agreements in written texts. British Journal of Educational Psychology, 74, 533550.

Matsumura, L. C., Patthey-Chavez, G. G., Valdés, R., \& Garnier, H. (2002). Teacher feedback, writing assignment quality and third grade students' revision in lower and higher achieving urban schools. The elementary School Journal, 103 (1), 3-25.

McCutchen, D. (2006). Cognitive factors in the development of children's writing. En Ch. A. MacArthur, S. Graham, \& J. Fitzgerald, (Eds.), Handbook of writing research (pp. 115-130). New York: The Guilford Press.

McCutchen, D. (1995). Cognitive processes in children's writing: Developmental and individual differences. Issues in Education: Contributions from Educational Psychology, 1, 123-160.

McCutchen, D., Francis, M., \& Kerr, S. (1997). Revising for meaning effects of knowledge and strategy. Journal of educational psychology, 89 (4), 667-676.

Perez, S. A. (2001). Revising During Writing in a Second Grade Classroom. Educational Research Quarterly, 25 (1), 27-32. 
Rijlaarsdan, G. \& van den Bergh, H. (2006). Writing process theory: a functional dynamic approach. En Ch. A. MacArthur, S. Graham, \& J. Fitzgerald, (Eds.), Handbook of writing research (pp. 41-53). New York: The Guilford Press.

Scardamalia, M., \& Bereiter, C. (1985). The development of dialectical processes in composition. En D. Olson, N. Torrance, \& A. Hildyard (Eds.), Literacy, language and learning: the nature and consequences of reading and writing (pp. 307-329). New York: Cambridge University Press.

Scott, B. J., \& Vitale, M. R., (2003). Teaching the writing process to students with LD. Intervention in School and Clinic, 38 (4), 220224.

Torrance, M., \& Galbraith, D. (2006). The processing demands of writing. En Ch. A. MacArthur, S. Graham, \& J. Fitzgerald, (Eds.), Handbook of writing research (pp. 67-80). New York: The Guilford Press.

Wallace, D. L., Hayes, J. R., Hatch, J. A., Miller, W., Moser, G., \& Silk, C. M. (1996). Better revision in eight minutes? Prompting first-year college writers to revise globally. Joumal of Educational Psychology, 88, 682-688.

Wong, B. Y. L. (1998). Reflections on current attainments and future directions in Writing Intervention Research in learning disabilities. En T. E. Scruggs, \& M. A. Martropieri (Eds.), Advances in learning and behavioral disabilities (pp. 127-149). Greenwich, CT: JAI Press Inc.

Wong, B. Y. L. (1999). Metacognition in Writing. En R. Gallimore, C. Bernheimer, D. McMillan, D. Speece, \& S. Vaughn (Eds.), Developmental perspectives on children with high incidence disabilities (pp. 183-198). N.J.: Erlbaum. 